\title{
Cardiac Risk Stratification of Neurosurgical Patients
}

\section{Aditya A Munshi}

Department of Neurological Surgery, Vickie and Jack Farber Institute for Neuroscience at Jefferson, Thomas Jefferson University, Philadelphia, PA 19107

\begin{abstract}
The role of an internal medicine physician in the perioperative setting includes the assessment of peri-operative risk, optimization of modifiable risk factors to decrease this risk and management of post-operative medical complications that may occur. Every patient undergoing surgery is at risk for procedural and anesthesia complications, in addition the patient is at risk of developing adverse medical events. Unlike surgical risk which is related to the procedure being performed and the risk of anesthesia, the factors affecting the medical risk are often modifiable. These modifiable risk factors form the principal basis of risk stratifying patients prior to surgery.

Neurosurgical patients pose certain unique challenges in the peri-operative setting and the pre-operative assessment forms a starting point in the prevention of not just post-operative cardiac complications but also thrombotic events and in reducing the overall morbidity and length of hospital stay.

In this chapter, we summarize our approach to the cardiac risk stratification of patients undergoing neurosurgery. We review recommendations from accepted guidelines and provide a step wise approach to the cardiac risk assessment of a patient undergoing elective surgery.
\end{abstract}

\section{INTRODUCTION}

The medical pre-operative evaluation has primarily comprised of assessing a patient's risk of developing major adverse cardiac events in the post-operative period. However, any patient undergoing surgery is exposed to a risk of several complications affecting different systems. These include pulmonary, cardiac, thrombotic, bleeding events, complications from the surgical procedure and anesthesia. The risks associated with anesthesia and the procedure itself are not modifiable and not discussed in this chapter. We cover the pre-operative assessment of a patient undergoing neurosurgery with a focus on cardiac risk assessment. Medical risks are modifiable in many cases and are affected by a patient's overall health, nutritional status, comorbid conditions and baseline activity level. The aim of this article shall be to review the most up-to-date guidelines and summarize our approach to a risk stratifying a patient undergoing neurosurgery from a cardiac standpoint.

\section{CARDIAC RISK STRATIFICATION OF NEUROSURGERY PATIENTS}

Around 235 million surgeries are performed globally every year. ${ }^{1}$ Cardiac events are the leading cause of post-operative complications, ${ }^{2}$ the risk of a patient developing cardiac complications depends on the patient's baseline risk. This is the principle of pre-op risk assessment and the aim of the pre-op cardiac assessment is to estimate this baseline risk and determine if the patient needs additional cardiac testing. The ACC/AHA guidelines form the cornerstone of pre-op assessment today. Per these guidelines, the risk stratification approach should consider the type of surgery, the urgency of the procedure being performed and clinical status of the patient. ${ }^{3}$ The guidelines define low risk procedures as those with a $<1 \%$ risk of major adverse cardiac events (MACE) based on combined patient and surgical characteristics. Whereas, the elevated risk group comprises of those with $\mathrm{a} \geq 1 \%$ risk of MACE3. The latest $\mathrm{ACC} /$ AHA guidelines, published in 2014, stratify procedures into these two categories. The approach to the patient depends on the category they fall into.

The timing of many neurosurgical procedures is urgent or emergent and this makes them high risk from the cardiac standpoint even if the patient has a low baseline risk. In addition, a large subset of the neurosurgical patient population is chronically ill and with multiple comorbid conditions that increases the risk of surgical complications. ${ }^{4,5}$

\section{CHOOSING PATIENTS WHO SHOULD UNDERGO A PRE-OPERATIVE ASSESSMENT:}

In choosing patients who should undergo a cardiac pre-operative assessment, certain factors need to be considered. Urgent and emergent surgery should not be delayed for a pre-operative evaluation. In these situations, the risk of delaying the procedure far outweighs the potential benefit of identifying underlying medical or cardiac problems. As an example, this situation often arises in neurosurgical patients who present with intracranial or spine pathology requiring immediate surgical intervention. Delaying surgery to assess cardiac risk in these patients would lead to devastating consequences.

The guidelines on pre-operative assessment are directed at patients undergoing elective surgery and do not recommend delaying surgery for assessment. ${ }^{3,6}$ These patients have a higher risk of cardiovascular adverse events even if their baseline risk level is low, risk indices are based on data from elective surgeries and are not accurate in patients undergoing urgent or emergent procedures and should not be used for these patients. Whenever possible, a thorough history and physical exam should be obtained for all patients. This should be to look for a history of 
bleeding events, serious drug allergies, and a history of medical comorbidities that could complicate the post-operative course.

For all other patients a step wise approach to cardiac risk stratification is outlined below.

\section{Step 1: Is the patient at very high risk for MACE?}

This group of patients includes patients with hemodynamically significant valvular heart disease, decompensated heart failure, high grade conduction blocks, supraventricular tachycardias with uncontrolled ventricular rate, malignant arrhythmias, symptomatic bradycardia, recent $\mathrm{MI}$ and unstable angina. ${ }^{7}$ They need to be referred to a specialist for workup and treatment of these conditions and should not undergo elective surgery without a consultation.

\section{Step 2: Is the patient at low ( $<1 \%)$ risk of MACE?}

The next step of the assessment is to use a risk estimation index to determine if the patient is at low risk of MACE $(<1 \%)$. There are several risk indices and Lee's Revised Cardiac Risk Index (RCRI) is one of he most widely used. Two additional risk indices based on the National Surgical Quality Improvement Program NSQIP may also be used. These are the Gupta scale and the NSQIP Myocardial Infarction and Cardiac Arrest (NSQIP MICA). Factors used in calculating the RCRI score are outlined in Table 1.

These scales are used to estimate the risk of MACE. $A \geq 1 \%$ risk of MACE puts the patient in the elevated risk category. Patients who are $<1 \%$ risk of MACE are considered low risk and no further cardiac testing is recommended. ${ }^{3}$

After the publication of the original RCRI, many estimates of cardiac events based on the RCRI points scored have been published. Pooled risk estimates showed that the event rates were higher than the original estimate. 6,8 These differences are discussed in the Canadian Cardiovascular Guidelines published in 2017 and are attributed to the use of troponin measurement and inclusion of emergency surgery patients in the more recent data. ${ }^{6}$ We follow the recommendations of the Canadian guidelines in

\section{Table 1. Lee's Revised Cardiac Risk Index}

\begin{tabular}{|c|c|}
\hline Factor & Points Assigned \\
\hline Ischemic heart disease $\mathrm{e}^{\#}$ & 1 \\
\hline History of heart failure* & 1 \\
\hline History of stroke or transient ischemic attack & 1 \\
\hline On insulin for diabetes & 1 \\
\hline Serum creatinine (>2.0 mg/dl) pre-operatively & 1 \\
\hline High-risk surgery ${ }^{\dagger}$ & 1 \\
\hline \multicolumn{2}{|c|}{$\begin{array}{l}\text { \# Defined as patient with a history of myocardial infraction (MI), positive exercise stress testing, ongoing chest pain, } \\
\text { presumed to be due to ishemia or use of nitrates or electrocardiogram with } Q \text { waves. }\end{array}$} \\
\hline \multicolumn{2}{|c|}{$\begin{array}{l}\text { * History of congestive heart failure, pulmonary edema, radiographic evidence of pulmonary vascular congestion, } \\
\text { paroxysmal dyspnea or physicial exam finding of } \$ 3 \text { gallop/bilateral rates. }\end{array}$} \\
\hline \multicolumn{2}{|c|}{${ }^{\dagger}$ High risk surgery was defined as intraperitoneal, intrathoracic, suprainguinal vascular procedures. } \\
\hline \multicolumn{2}{|c|}{$\begin{array}{l}\text { Modified version of index published by Lee TH et al. Derivation prospective validation of a simple index } \\
\text { for prediction of cardiac risk of major noncardiac surgery. Circulation. } 199 \text { Sept 7; 10(10):1043-9 }\end{array}$} \\
\hline
\end{tabular}

\section{Table 2. Risk Estimates from The Revised Cardiac Risk Index}

\begin{tabular}{|l|c|c|}
\hline $\begin{array}{l}\text { RCRI score } \\
\text { (Points) }\end{array}$ & $\begin{array}{c}\text { Risk estimate (\%) and 95\% Cl } \\
\text { based on original data* }\end{array}$ & $\begin{array}{r}\text { Risk estimate and 95\% Cl based } \\
\text { on pooled data3 }\end{array}$ \\
\hline 0 & $0.4 \%[0.05 \%-1.5 \%]$ & $3.9 \%[2.8 \%-5.4 \%]$ \\
\hline 1 & $0.9 \%[0.3 \%-2.1 \%]$ & $6.0 \%[4.9 \%-7.4 \%]$ \\
\hline 2 & $6.6 \%[3.9 \%-10.3 \%]$ & $10.1 \%[8.1 \%-12.6 \%]$ \\
\hline$\geq 3$ & $11.0 \%[5.8 \%-18.4 \%]$ & $15.0 \%[11.1 \%-20.0 \%]$ \\
\hline
\end{tabular}

* Estimates from the original published data by Lee TH et al. Derivation and prospective validation of a simple index for prediction of cardiac risk of major noncardiac surgery. Circulation. 1999 Sep 7; 100(10):1043-9

\# Risk estimates from Duceppe et al. Canadian Cardiovascular Society Guidelines on Perioperative Cardiac Risk Assessment and Mamagement for Patients Who Undergo Noncardiac Surgery. These estimates were based on external validations published after the original study by Lee TH et al (1999).

\section{Table 3. Metabolic Equivalents (METS) of some common activities}

\begin{tabular}{|l|c|}
\hline Activity & METS \\
\hline Resting state & 1 MET \\
\hline Cooking & $2-3$ METS \\
\hline Car Driving & 2 METS \\
\hline Walking (3 miles/hr or 5 km/hr) & 3 METS \\
\hline Climb Stairs & $4-5$ METS \\
\hline Snow Shoveling & 5 METS \\
\hline Running (8 miles/hr or 13 km/hr) & 13 METS \\
\hline $\begin{array}{l}\text { \# For a 70 kg individual. Values are approximate values based on data published by Jette et al. in Metabolic } \\
\text { equivalents (METS) in exercise testing, exercise prescription, and evaluation of functional capacity. } \\
\text { Clin Cardiology 1990 Aug;13(8):555-65 }\end{array}$ \\
\hline
\end{tabular}


using a RCRI score of $\geq 1$ point and do not recommend using the estimated percent risk to classify patients under the elevated risk category (Table 2).

\section{Step 3: Assess Functional Capacity}

Under the AHA guidelines, the next step in assessing patients under the elevated risk category is to estimate functional capacity. Self-reported functional capacity is the most widely used method of estimation. The patient is asked what level of exertion they can tolerate without experiencing symptoms. Metabolic equivalents (METS) of many common activities are outlined in Table 3 .

The Duke activity status index (DASI) is a standardized tool for estimating functional capacity. ${ }^{9}$ A study comparing the DASI, self-reported functional capacity and cardiopulmonary exercise testing showed that only scores on DASI correlated with cardiac events in the post-op period..$^{10}$ Therefore, if functional capacity is included in the pre-op assessment, it is recommended that a standardized estimation tool like the DASI be used.

Per the approach outline in the ACC/AHA guidelines, patients at elevated risk, tolerating >4 METS without symptoms do not need additional cardiac testing. Whereas, patients at elevated risk with an unknown functional capacity or not able to reach 4 METS require additional cardiac testing. Various modalities for cardiac testing in these patients is outlined below.

\section{CARDIAC TESTING MODALITIES}

\section{Stress Testing}

The ACC/AHA guidelines recommend stress testing (exercise or pharmacologic with dobutamine stress echocardiography (DSE) or myocardial perfusion imaging (MPI) in patients at elevated risk for noncardiac surgery and have an unknown or poor (< 4 METS) functional capacity if it will change management. ${ }^{3}$

Routine stress testing just because a patient is undergoing noncardiac surgery is not recommended. ${ }^{11} \mathrm{~A}$ stress test showing a large area of ischemic myocardium or multiple reversible defects on MPI is associated with a higher incidence of post-op death from cardiac causes or non-fatal myocardial infarction. ${ }^{12,13}$
Additionally, findings from several single center studies have shown that a negative stress test has a high negative predictive value. This is useful for patients who are moderate risk but limits the utility of stress testing in the highest risk patients. ${ }^{14,15}$

\section{Resting Echocardiography:}

Echocardiography to assess left ventricular function is recommended if the patient has dyspnea of unknown etiology, if they patient has heart failure and there is a change in their clinical condition or if a patient with stable heart failure has not had an echocardiograph in one year. ${ }^{3,16}$

Echocardiography to assess valvular function should be performed if a patient has known valvular disease and presents with a change clinically or if the patient presents with clinical signs of moderate or severe valvular heart disease. 3,16

The Canadian guidelines published in 2017 favor biomarker testing and recommend a move away from resting echocardiography for pre-op testing, this is based on evidence showing that biomarker testing is superior to echocardiography in predicting adverse perioperative outcomes. ${ }^{17} \mathrm{It}$ is suggested that a resting echocardiogram should not be performed as a substitute to stress testing or biomarker measurement for high risk patients. However, resting echocardiography should be performed in patients suspected of having systolic heart failure to evaluate their left ventricular ejection fraction. It should also be done in patients with suspected moderate or severe valvular heart disease if they have not had an echocardiogram in the last one year or there has been a significant change in their clinical status since the last evaluation.

\section{Role of pre-operative electrocardi- ography (ECG):}

The ACC/AHA guidelines recommend obtaining an electrocardiogram in patients undergoing intermediate or high risk noncardiac surgery if they have a history of structural heart disease, arrhythmias, or vascular disease (includes CAD, stroke, TIA, or peripheral arterial disease). ECGs are not recommended for low risk surgery. Routine ECGs based on age cut-offs are also not recommended. ${ }^{3,18}$
The electrocardiogram is often performed in patients undergoing surgery based on arbitrary age cut-offs. A resting echocardiogram is useful in the detection of arrhythmias and to compare to baseline for patients with known CAD, peripheral vascular disease, cerebrovascular disease or ischemic heart disease. ${ }^{19,20}$ However, its utility is significantly limited when a patient's baseline ECG is unknown or if nonspecific abnormalities are found on the ECG. These limitations make it less useful in diagnosing asymptomatic CAD in patients undergoing noncardiac surgery.

\section{THE CANADIAN CARDIOVASCULAR SOCIETY GUIDELINES}

The Canadian guidelines published in 2017 changed long standing recommendations on cardiac pre-operative evaluation and advocated a move towards biomarker measurement and post-operative troponin level testing. ${ }^{6}$ We summarize the main recommendations from the paper below.

- Measurement of biomarkers (proBNP or NT-proBNP) prior to surgery in patients who are $>65$ years of age or have RCRI $\geq 1$ or are 45-64 years old with cardiac comorbidities.

- Advise against performing resting echocardiography, coronary computed tomography angiography, stress testing or cardiopulmonary exercise testing or nuclear imaging as a part of perioperative risk assessment.

- Recommend daily troponin measurement for patients with elevated biomarker (pro-BNP or NT-proBNP) or if biomarkers are not measured but they score 1 or more points on the RCRI scale.

Additionally, The Canadian Cardiovascular Society guidelines recommend the following for continuing or initiating medications in the perioperative period.

- Acetylsalicylic Acid (ASA) should not be continued or initiated for cardiovascular protection or prevention. Exceptions include patients who had a recent coronary stent placement or will undergo carotid endarterectomy 
- B-blockers or $\alpha 2$-agonist initiation is not recommended in the 24 hours prior to surgery.

- Recommends holding angiotensinconverting enzyme inhibitors (ACEI) and angiotensin II receptor blockers 24 hours prior to surgery.

- If a patient develops myocardial infarct or injury in the post-operative period, a statin and ASA should be started.

\section{CONCLUSION}

Our article summarizes existing guidelines into a stepwise approach that provides a picture of our current practice as a hospitalist group providing perioperative care to neurosurgical patients. We attempt to highlight appropriate indications and use criteria for cardiac testing methods. Neurosurgical patients, both spine and intracranial can have high morbidity and need careful perioperative care. Keeping this in mind, it is even more imperative that appropriate testing be performed when indicated and unnecessary testing be avoided.

\section{REFERENCES}

1. Weiser TG, Haynes AB, Molina G, et al. Estimate of the global volume of surgery in 2012: an assessment supporting improved health outcomes. Lancet 2015;385 Suppl 2:S11.

2. Lee LKK, Tsai PNW, Ip KY, Irwin MG. Pre-operative cardiac optimisation: a directed review. Anaesthesia 2019;74 Suppl 1:67-79.

3. Fleisher LA, Fleischmann KE, Auerbach AD, et al. 2014 ACC/AHA guideline on perioperative cardiovascular evaluation and management of patients undergoing noncardiac surgery: a report of the American College of Cardiology/American Heart Association Task Force on practice guidelines. J Am Coll Cardiol 2014;64:e77-137.

4. Bapat S, Luoma AM. Current UK practice of pre-operative risk assessment prior to neurosurgery. Br J Neurosurg 2016;30:195-9.
5. Quinn TD, Brovman EY, Aglio LS, Urman RD. Factors associated with an increased risk of perioperative cardiac arrest in emergent and elective craniotomy and spine surgery. Clin Neurol Neurosurg 2017;161:6-13.

6. Duceppe E, Parlow J, MacDonald P, et al. Canadian Cardiovascular Society Guidelines on Perioperative Cardiac Risk Assessment and Management for Patients Who Undergo Noncardiac Surgery. Can J Cardiol 2017;33:17-32.

7. Fleisher LA, Beckman JA, Brown KA, et al. 2009 ACCF/AHA focused update on perioperative beta blockade incorporated into the ACC/AHA 2007 guidelines on perioperative cardiovascular evaluation and care for noncardiac surgery: a report of the American college of cardiology foundation/American heart association task force on practice guidelines. Circulation 2009;120:e169-276.

8. Lee $\mathrm{TH}$, Marcantonio ER, Mangione CM, et al. Derivation and prospective validation of a simple index for prediction of cardiac risk of major noncardiac surgery. Circulation 1999;100:1043-9.

9. Hlatky MA, Boineau RE, Higginbotham MB, et al. A brief self-administered questionnaire to determine functional capacity (the Duke Activity Status Index). Am J Cardiol 1989;64:651-4

10. Wijeysundera DN, Pearse RM, Shulman MA, et al. Assessment of functional capacity before major non-cardiac surgery: an international, prospective cohort study. Lancet 2018:391:2631-40.

11. Carliner NH, Fisher ML, Plotnick GD, et al. Routine preoperative exercise testing in patients undergoing major noncardiac surgery. Am J Cardiol 1985;56:51-8.

12. Etchells E, Meade M, Tomlinson G, Cook D. Semiquantitative dipyridamole myocardial stress perfusion imaging for cardiac risk assessment before noncardiac vascular surgery: a meta-analysis. J Vasc Surg 2002;36:534-40.

13. Shaw LJ, Eagle KA, Gersh BJ, Miller DD. Meta-analysis of intravenous dipyridamolethallium-201 imaging (1985 to 1994) and dobutamine echocardiography (1991 to 1994) for risk stratification before vascular surgery. J Am Coll Cardiol 1996;27:787-98.
14. Kertai MD, Boersma E, Bax JJ, et al. A metaanalysis comparing the prognostic accuracy of six diagnostic tests for predicting perioperative cardiac risk in patients undergoing major vascular surgery. Heart 2003;89:1327-34.

15. Picano E, Bedetti G, Varga A, Cseh E. The comparable diagnostic accuracies of dobutamine-stress and dipyridamole-stress echocardiographies: a meta-analysis. Coron Artery Dis 2000:11:151-9.

16. American College of Cardiology Foundation Appropriate Use Criteria Task F, American Society of E, American Heart A, et al. ACCF/ ASE/AHA/ASNC/HFSA/HRS/SCAI/SCCM/ SCCT/SCMR 2011 Appropriate Use Criteria for Echocardiography. A Report of the American College of Cardiology Foundation Appropriate Use Criteria Task Force, American Society of Echocardiography, American Heart Association, American Society of Nuclear Cardiology, Heart Failure Society of America, Heart Rhythm Society, Society for Cardiovascular Angiography and Interventions, Society of Critical Care Medicine, Society of Cardiovascular Computed Tomography, and Society for Cardiovascular Magnetic Resonance Endorsed by the American College of Chest Physicians. J Am Coll Cardiol 2011;57:1126-66.

17. Park SJ, Choi JH, Cho SJ, et al. Comparison of transthoracic echocardiography with $\mathrm{N}$-terminal pro-brain natriuretic Peptide as a tool for risk stratification of patients undergoing major noncardiac surgery. Korean Circ J 2011;41:505-11.

18. Gold BS, Young ML, Kinman JL, Kitz DS, Berlin J, Schwartz JS. The utility of preoperative electrocardiograms in the ambulatory surgical patient. Arch Intern Med 1992;152:301-5.

19. Jeger RV, Probst C, Arsenic R, et al. Longterm prognostic value of the preoperative 12-lead electrocardiogram before major noncardiac surgery in coronary artery disease. Am Heart J 2006;151:508-13.

20. Payne CJ, Payne AR, Gibson SC, Jardine AG, Berry C, Kingsmore DB. Is there still a role for preoperative 12-lead electrocardiography? World J Surg 2011;35:2611-6. 